\title{
Activity and interaction of trimethoprim and sulphamethoxazole against Escherichia coli
}

\author{
D. GREENWOOD ${ }^{1}$ AND F. O'GRADY' \\ From the Department of Bacteriology, St Bartholomew's Hospital, London ECIA $7 B E$
}

SYNOPSIS The activity of trimethoprim (TMP) and sulphamethoxazole (SMX), alone and in combination, against a sensitive strain of Escherichia coli was investigated in turbidimetric systems. In a static system in which the conditions of exposure of bacteria to drug resembled those of conventional minimum inhibitory concentration (MIC) titrations, both TMP and SMX exhibited antibacterial activity at concentrations well below the conventionally determined MIC, but regrowth $\stackrel{乛}{\mathrm{~N}}$ occurred at these concentrations during the overnight incubation period due to the emergence of adaptively resistant bacteria. Tests of combined drug action in the static turbidimetric system $\rightarrow$ revealed even more synergic interaction than was apparent in conventional MIC tests. It is suggested that an important component of overall synergic interaction is the mutual suppression of adaptive 'resistance' to the other agent.

Studies in an in vitro model which simulates the hydrokinetic features of the urinary bladder showed that concentrations of TMP and SMX below the conventionally determined MIC inhibite the growth even of extremely dense bacterial populations so long as the concentration of drug wags maintained. The response of cultures exposed to combinations of TMP and SMX in this systeen was so dominated by the effect of TMP that no synergic interaction with SMX was noted at con? centrations of the drugs which are achievable in urine.

Trimethoprim (TMP) and sulphamethoxazole (SMX) show marked synergy in vitro and the combination has been widely and successfully used for the treatment of a variety of infections. Not unnaturally, it has been generally supposed that the therapeutic success of the combination is a consequence of the synergic interaction of its components. However, TMP is an extremely potent antibacterial agent in its own right, and doubts have been expressed about the role of the sulphonamide in the therapeutic effects of the combination.

As a contribution to this on-going argument we have investigated the response of Escherichia coli to TMP and SMX, alone and in combination, in turbidimetric systems in which the response of cultures can be continuously monitored. One of these systems simulates several important features of the conditions in which bacteria and antibacterial agents interact in the urinary bladder, the site of an infection frequently treated with the combination.

'Present address: Department of Microbiology, University of Nottingham, City Hospital, Nottingham NG5 1 PH

Received for publication 22 July 1975

\section{Material and Methods}

A TMP- and SMX-sensitive strain of $E$. coli (designated ECSA 1), originally isolated from infected urine, was used throughout.

Sulphamethoxazole was obtained as pure powder

from Roche Products Ltd. Trimethoprim lactate was obtained from Burroughs Wellcome Ltd. Solutions of the agents were freshly prepared in sterile distilled water as required.

Wellcotest broth (Wellcome Reagents Ltd), prepared according to the manufacturer's instructions, was used as growth medium.

Minimum inhibitory concentrations (MIC) of TMP and SMX were estimated, using serial doubling N dilutions of the agents in broth with a bacterial inoculum of $\mathrm{ca} 10^{5}$ organisms per ml derived from a 4-hour broth culture.

'STATIC' SYSTEM

Turbidimetric experiments were carried out using a device in which the opacity of 12 independent bacterial cultures can be simultaneously monitored continuously (Mackintosh et al, 1973a). In these 
experiments $25 \mathrm{ml}$ volumes of broth containing appropriate concentrations of antibacterial agent were inoculated with 1 drop of an overnight broth culture of bacteria and incubated in the light paths of the turbidity monitoring device. Each culture was mixed using a magnetic stirrer.

\section{DILUTION SYSTEM}

Details of the design of the 'bladder' model and its application to the study of antibiotic effects have been described elsewhere (Mackintosh et al, 1973b; O'Grady et al, 1973). In the model, a fully grown broth culture of bacteria is diluted with fresh broth at a rate equivalent to the normal diurnal ureteric urine flow rate $(1 \mathrm{ml}$ per minute) and at preset intervals (1 hour in the present series of experiments) a 'micturition' episode empties the 'bladder' leaving a residual volume of $20 \mathrm{ml}$. The culture is mixed by a stainless steel paddle and the turbidity is continuously monitored photometrically.

\section{Results}

The MICs of TMP and SMX for the E. coli strain, as judged by conventional titration in broth using a bacterial inoculum of $c a 10^{5}$ organisms per $\mathrm{ml}$, were 1 and $10 \mu \mathrm{g}$ per $\mathrm{ml}$ respectively.

\section{STATIC SYSTEM}

Continuous opacity records of cultures exposed to TMP and SMX are shown in figures 1 to 3 . In experiments simulating conventional MIC titrations, in which 1 drop of an overnight broth culture was added to drug-containing broth, both TMP (fig 1) and SMX (fig 2) were found significantly to suppress growth at sub-MIC concentrations, but regrowth occurred during the overnight period. In both cases an increase in opacity occurred during the first few hours' exposure to concentrations at or above a quarter of the conventionally determined MIC (MIC/4). This was more marked with SMX and

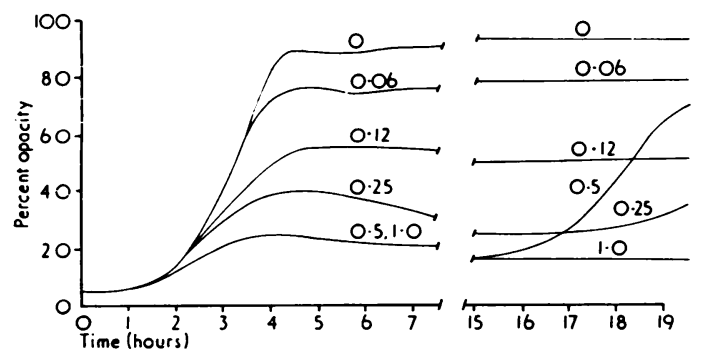

Fig 1 Continuous opacity records of an $\mathrm{E}$. coli strain in the presence of various concentrations $(\mu \mathrm{g} / \mathrm{ml})$ of TMP.

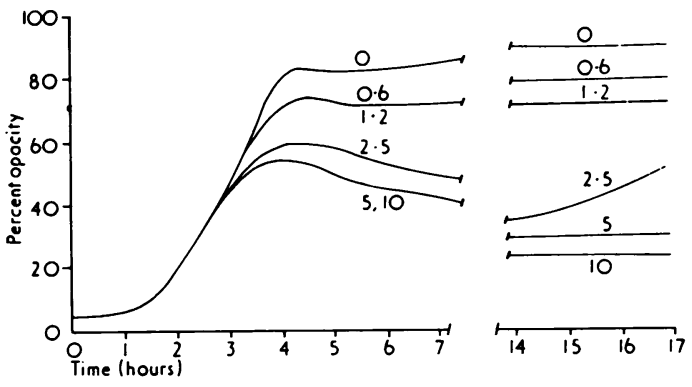

Fig 2 Continuous opacity records of an $\mathrm{E}$. coli strain in the presence of various concentrations $(\mu \mathrm{g} / \mathrm{ml})$ of $S M X$.

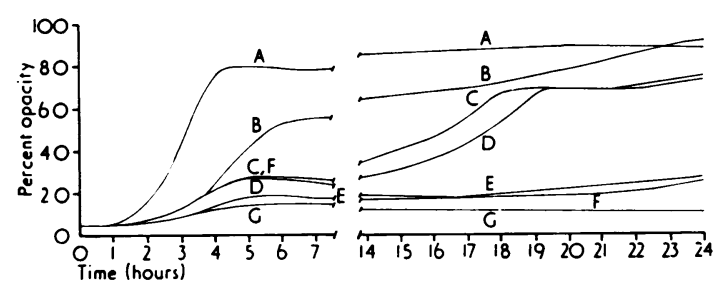

Fig 3 Continuous opacity records of an $\mathrm{E}$. coli strain in the presence of (per $\mathrm{ml}$ ): (A) no antibiotic; (B) $0.008 \mu \mathrm{g}$ $T M P+0.6 \mu g S M X$; (C) $0.008 \mu g T M P+1.2 \mu g S M X$; (D) $0.015 \mu g$ TMP $+0.6 \mu g S M X$; (E) $0.06 \mu g$ TMP + $0.6 \mu g S M X$; (F) $0.03 \mu g T M P+1.2 \mu g S M X$; (G) $0.06 \mu g T M P+1.2 \mu g S M X$.

was followed by a prolonged phase in which the opacity declined. Cultures regrowing during the overnight period after initial suppression of growth by either TMP or SMX were shown to be able to grow immediately on subinoculation into fresh broth containing the same drug concentration (that is, they had become 'resistant' to that concentration), but subsequent testing of these cultures after passage through drug-free broth showed that the 'resistance' was unstable. Subcultures of the regrowing cultures onto nutrient agar plates excluded the possibility of growth being due to contaminants.

When combinations of TMP and SMX were examined in the same way, the lowest concentrations that produced a slight but measurable retardation of growth were $0.008 \mu \mathrm{g}$ TMP $+0.6 \mu \mathrm{g} \mathrm{SMX} \mathrm{per} \mathrm{ml}$ (ca MIC/120 and MIC/16 respectively; fig 3B). Doubling the concentration of either component $(0.008 \mu \mathrm{g}$ TMP $+1.2 \mu \mathrm{g}$ SMX per ml; fig $3 \mathrm{C}$, or $0.015 \mu \mathrm{g}$ TMP $+0.6 \mu \mathrm{g}$ SMX per ml; fig 3D) markedly suppressed growth for about 12 hours, but regrowth subsequently occurred. The lowest concentrations of the combination able to suppress growth overnight were $0.06 \mu \mathrm{g}$ TMP $+1.2 \mu \mathrm{g} \mathrm{SMX}$ per $\mathrm{ml}$ (ca MIC/15 and MIC/8 respectively; fig 3G). 
If the concentration of either component was halved, slow regrowth occurred during the overnight period (fig 3E, F), but halving the concentration of TMP allowed more initial increase in opacity than halving the concentration of SMX (fig 3F).

\section{DILUTION SYSTEM}

The general form of bacterial growth in the 'bladder' model, using Wellcotest broth, was found to be somewhat different from that previously reported for experiments where a 'complete' broth was used (O'Grady et al, 1973; Greenwood and O'Grady, 1974). The initial lag was much shorter with Wellcotest broth than with complete medium, so that opacity changes due to bacterial growth occurred during the first hour of dilution and the culture more rapidly attained the 'fluctuating equilibrium' state in which the same cycle of opacity changes occurs between each 'micturition' episode (O'Grady et al, 1973). The form of the normal growth/dilution response of $E$. coli in Wellcotest broth is shown in figure 4. The addition of a single pulse of SMX, to achieve an initial concentration of $500 \mu \mathrm{g}$ per ml, immediately after the fourth hourly 'micturition' had no effect on this normal growth pattern.

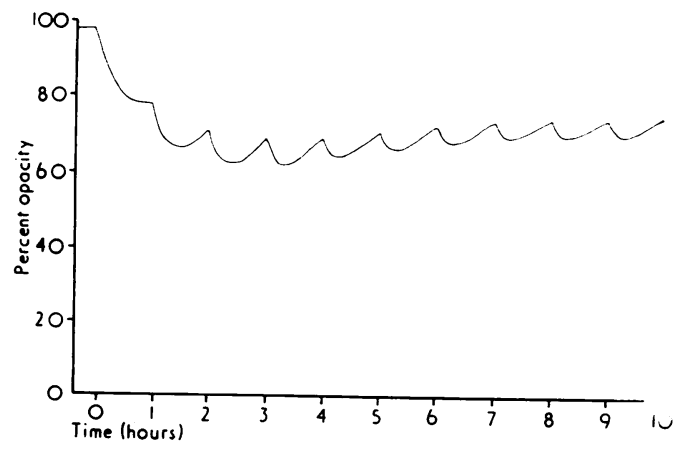

Fig 4 Typical opacity record obtained in the bladder model using antibiotic-free Wellcotest broth.

When a single pulse of TMP, to achieve an initial concentration of $50 \mu \mathrm{g}$ per $\mathrm{ml}$, was introduced into the system after the fourth 'micturition', a depressant effect on the growth of the culture was immediately seen (fig 5) and recovery did not occur until about 6 hours after the introduction of the pulse ( 3 hours after dilution and discharge had reduced the concentration of TMP to below the MIC). A mixture of $500 \mu \mathrm{g}$ SMX and $50 \mu \mathrm{g}$ TMP per ml, given as a pulse in the same way, produced an opacity trace identical with that obtained with TMP alone.

Further experiments were carried out in which TMP and SMX were infused with the diluent broth

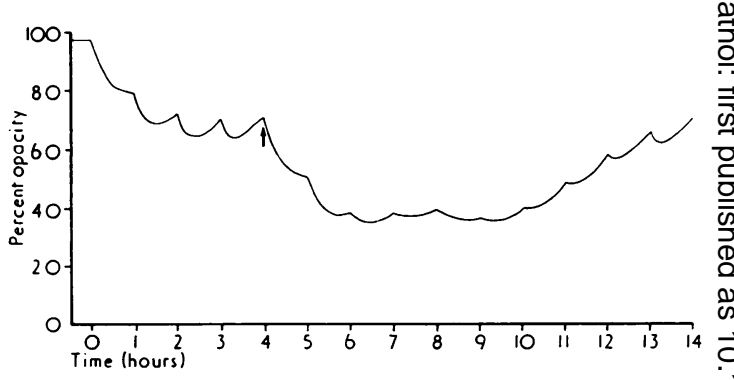

Fig 5 Continuous opacity record obtained using the bladder model. A single pulse of TMP, to achieve an initial concentration of $50 \mu \mathrm{g} / \mathrm{ml}$, was added after 4 cycles of dilution and 'micturition'.

rather than given as a single pulse. When SMX was $\vec{\sim}$ continuously infused it had no effect on the normal ${ }^{N}$ growth pattern for the first 2 hours, but the opacity subsequently fell progressively throughout the $\vec{T}$ infusion period even when the SMX concentration $\mathbb{D}$ was only $5 \mu \mathrm{g}$ per $\mathrm{ml}$ (fig 6), less than the con-을 ventionally determined MIC.

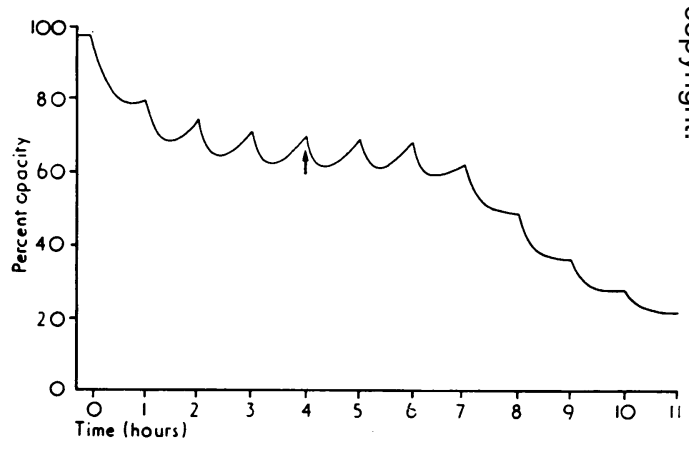

Fig 6 Continuous opacity record obtained using the bladder model. After 4 cycles of dilution and 'micturition', dilution was continued with broth containing $5 \mu \mathrm{g} S \mathrm{MX} / \mathrm{ml}$.

In a similar manner, a TMP concentration in the diluent broth of $0.25 \mu \mathrm{g}$ per $\mathrm{ml}$ (MIC/4) produced $\mathrm{a} \cong$. progressive fall in opacity throughout the dilution period. With this agent, the fall in opacity began during the first hour of infusion (fig 7). When SMXO $(5 \mu \mathrm{g}$ per $\mathrm{ml})$ and TMP $(0.25 \mu \mathrm{g}$ per $\mathrm{ml})$ were infused $\omega$ together, the subsequent opacity trace was identical with that found with TMP alone.

In order to study the regrowth pattern of the cultures following prolonged exposure to these $e^{\text {? }}$ agents, SMX and TMP alone and in combination were infused into the 'bladder' for a period of $8 \frac{\mathrm{O}}{\mathrm{P}}$ hours, after which dilution was continued with? drug-free broth. In one set of experiments concen $\frac{\mathbb{D}}{2}$ 


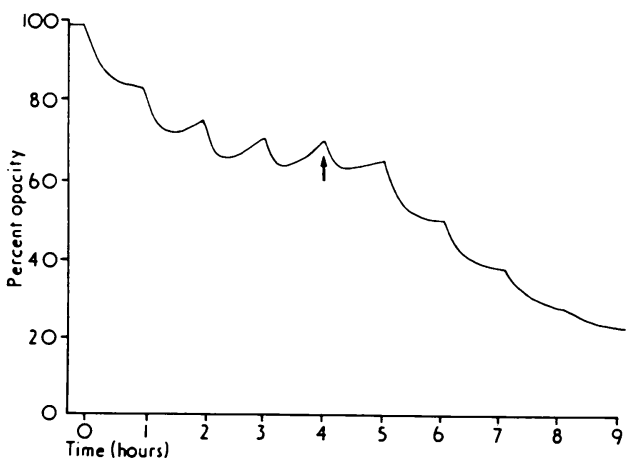

Fig 7 Continuous opacity records obtained using the bladder model. After 4 cycles of dilution and 'micturition' dilution was continued with broth containing $0.25 \mu \mathrm{g}$ $T M P / m l$.

trations of $5 \mu \mathrm{g} \mathrm{SMX}$ and $0.25 \mu \mathrm{g}$ TMP per ml were used; in another set, $100 \mu \mathrm{g}$ SMX and $5 \mu \mathrm{g}$ TMP per $\mathrm{ml}$ were employed. When either agent was used alone, the higher concentration had a somewhat greater depressant effect on the opacity of the culture, and regrowth was correspondingly delayed when the infusion was continued with drug-free broth. When the combination of SMX and TMP was used, the response pattern was very similar to that obtained with TMP alone, although an increase in the recovery time was observed at the lower concentration levels. The times elapsing before regrowth was detectable in the photometric system following the switch to drug-free broth are shown for the two agents alone and in combination in the table.

\begin{tabular}{|c|c|c|}
\hline $\begin{array}{l}\text { Antibacterial } \\
\text { Agent }\end{array}$ & $\begin{array}{l}\text { Concentration } \\
\text { ( } \mu \text { g per } m l) \\
\text { in Infusion }\end{array}$ & $\begin{array}{l}\text { Time (hr) to Regrowth } \\
\text { after Switch to } \\
\text { Drug-free Broth }\end{array}$ \\
\hline SMX & 5 & $1 \cdot 5$ \\
\hline TMP & 0.25 & 1.5 \\
\hline $\mathbf{S M X}+\mathbf{T M P}$ & $5+0.25$ & 3 \\
\hline SMX & 100 & 3 \\
\hline TMP & 5 & 6 \\
\hline $\mathbf{S M X}+\mathbf{T M P}$ & $100+5$ & 6 \\
\hline
\end{tabular}

Table Comparison of the ability of cultures of $\mathrm{E}$. coli to recover following exposure to SMX and/or TMP for

8 hours, in the bladder model

SMX = sulphamethoxazole; TMP $=$ trimethoprim

\section{Discussion}

Continuous turbidimetric monitoring of the response of $E$. coli to TMP or SMX in a static system revealed that concentrations of the agents below the conventionally determined MIC had a marked inhibitory effect on the cultures, but that regrowth occurred during the overnight period due to the emergence of facultatively resistant cells. When cultures were exposed to the TMP/SMX combination, prolonged suppression of growth was obtained at very low drug concentrations (MIC/120 TMP + MIC/8 SMX or MIC/60 TMP + MIC/16 SMX), and overnight suppression of growth was achieved at minimum concentrations of MIC/15 TMP + MIC/8 SMX. These results indicate that the combination is even more synergic than conventional MIC titrations (usually read at a fixed point in time after overnight incubation) suggest; that MIC titration results include a factor attributable to 'adaptive' resistance; and that an important component of the synergic effect may be the mutual suppression of adaptive variants to the other agent.

Tests of antibacterial activity of sulphonamides are well known to be markedly affected by the size of the bacterial inoculum, yet they have been successfully used for many years in the treatment of urinary tract infection where the bacterial numbers with which the agent may have to cope are frequently extremely high. Thus it was of particular interest to study the response of dense bacterial cultures to SMX in the simulated bladder conditions. A single pulse of SMX, even at as high a level as $500 \mu \mathrm{g}$ per $\mathrm{ml}$, had no effect on the normal bacterial growth pattern in the bladder model. However, when the agent was infused continuously an effect was achieved, after a lag of several hours, even if the concentration of SMX in the infusion was as low as $5 \mu \mathrm{g}$ per $\mathrm{ml}(\mathrm{MIC} / 2)$. These results support the view that the inoculum effect seen with sulphonamides is due to the presence of an intracellular folate pool which continues to supply the needs of the population until bacterial growth dilutes the pool to below a critical level, and explain why therapy with this group of agents (many of which are excreted into the urine slowly over a long period) is often successful despite the dense bacterial populations involved.

Similar findings were made using TMP. In the case of this agent, the inoculum effect is not so marked, and in the model this was reflected by a more rapid, sharper decline in bacterial numbers when TMP was continuously infused. A single large pulse of TMP had a transient effect, and continuous infusion of $\mathrm{MIC} / 4$ produced a prolonged decline in bacterial numbers.

Synergy between TMP and SMX was examined in the model by exposing cultures to various combinations of single pulses of different sizes and by infusing the two agents for different times. In no case did we demonstrate any substantial synergy, as measured by prolongation of the recovery time, except in the case of cultures exposed to sub-MIC concentrations of the two agents for 8 hours (table). This strongly suggests that as long as TMP is present 
at inhibitory levels, the contribution of SMX to the overall effect is very small. TMP does not allow as much residual bacterial growth as SMX (cf figs 1 and 2 ), presumably because its effect is to trap folate in the dihydrofolate form (Hitchings, 1973) and folate starvation occurs as much by this route as by dilution by cell division. In contrast, sulphonamides await dilution of folate by division to exert their effect. Consequently, as long as TMP is present in inhibitory concentration, the intracellular folate pool, in the form of dihydrofolate, may not become sufficiently dilute for sulphonamides to exert any effect at all even on prolonged exposure. Indeed, when looked at in this way TMP may be considered to antagonize the action of sulphonamides.

These considerations may have important therapeutic implications, for the synergy readily demonstrable in conventional in vitro tests may operate in vivo only in circumstances in which subinhibitory concentrations of TMP occur. Thus synergy may play little or no part in the eradication of bacterial cystitis but may be important in, for example, systemic or respiratory tract infection where concentrations of the agents at the site of infection may be low.
We thank the Governors and Joint Research Boardo of St. Bartholomew's Hospital for a grant towards the cost of this work.

\section{References}

Greenwood, D. and O`Grady, F. (1974). The comparative $\frac{\overline{\mathcal{S}}}{\bar{S}}$ performance of beta-lactam antibiotics against ampicillin sensitive Escherichia coli in conditions simulating those of the infected urinary bladder. Brit. J. exp. Path., 55, 245-250.6

Hitchings, G. H. (1973). The biochemical basis for the antimicrobial activity of Septrin. In Trimethoprim/sulpha methoxazole in Bacterial Infections, edited by L. S. Bern二 stein and A. J. Salter, pp 7-16. Churchill Livingstone Edinburgh and London.

Mackintosh, I. P., O'Grady, F., Greenwood, D., Watsono B. W., Crichton, T. C., Piper, R., and Ferrer, A. (1973a)in A twelve channel bacterial growth monitoring system iv Bio-med. Engng, 8, 514-515 and 526.

Mackintosh, I. P., Watson, B. W., and O'Grady, F. (1973b) $\vec{\sigma}$ Development and further applications of a simple turbidity cell for continuously monitoring bacterial growth. Phys.음 in Med. Biol., 18, 265-271.

O'Grady, F., Mackintosh, I. P., Greenwood, D., and Watson, $\vec{T}$ B. W. (1973). Treatment of 'bacterial cystitis' in fully automatic mechanical models simulating conditions of bacterial growth in the urinary bladder. Brit. J. exp. Path. ฏ) 54, 283-290. 